\title{
Charakterisierung von Lebergewebe mittels impedimetrischer Mikrosensoren
}

Alice Fischerauer, Carolin Grill, Maximiliane Würtheim, Gerhard Fischerauer Lehrstuhl für Mess- und Regeltechnik, Universität Bayreuth, 95440 Bayreuth 0921 55-7231 (Telefon), 0921 55-7235 (Fax), mrt@uni-bayreuth.de

\section{Einleitung}

Die schnelle Charakterisierung von organischem Gewebe ist in der Medizin wie auch in der Lebensmittelindustrie von hohem Interesse. Hier hat die Impedanzspektroskopie Eingang gefunden in Untersuchungen in der Transplantationsdiagnostik [1, 2, 3] und der Überwachung von Fleisch [4], da sie eine wenig aufwändige Erfassung großer Datenmengen mit hoher Informationsdichte über beliebig lange Zeiträume erlaubt [1]. Um ein Objekt beurteilen zu können, muss dieses häufig über einen längeren Zeitraum beobachtet werden, da vor allem Änderungen in der Impedanz ausgewertet werden. Diese Arbeit befasst sich mit der Frage, ob mittels planarer, auf Interdigitalelektroden (IDE) basierender Dünnschichtsensoren durch eine relativ kurze Messung bereits Informationen über den Zustand von Lebergewebe gewonnen und verschiedene Gewebesorten unterschieden werden können und welche Einflussgrößen dabei relevant sind. Letztendliches Ziel ist die Entwicklung von Mikrosensoren, die nur geringe Materialproben oder minimale Eingriffe in das Organ benötigen.

\section{Sensorgeometrie und Modellbildung}

Die verwendeten Sensoren bestehen aus planaren, interdigitalen Kammelektroden mit 150 Fingerpaaren der Länge $4 \mathrm{~mm}$, der Fingerbreite $10 \mu \mathrm{m}$ und der Fingerhöhe $100 \mathrm{~nm}$ (Bild 0, 1). An Hand ihres vertikalen Schichtaufbaus können die Sensoren in sechs Gruppen eingeteilt werden, die sich durch das Trägermaterial (Glas [herkömmlicher Objektträger] oder Silizium) und das Vorhandensein von Passivierungsschichten aus $\mathrm{SiO}$ oder $\mathrm{SiO}_{2}$ auf Substrat und Elektroden unterscheiden (Tabelle 1). Glas wurde als Substrat gewählt, weil es wegen seiner Kostengünstigkeit auch Einwegsensoren ermöglich, Silizium, weil damit die Integration zu „Lab-on-a-chip“-Systemen denkbar ist.

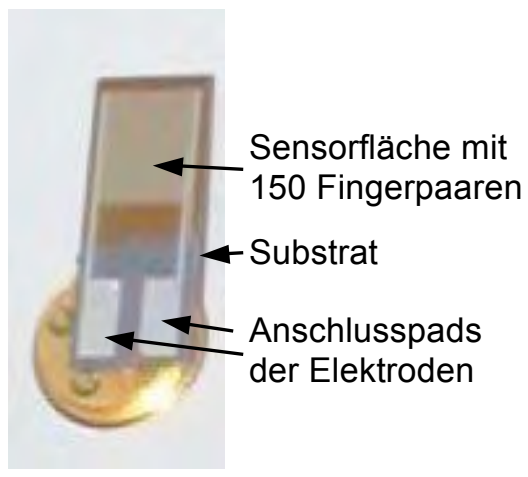

Bild 0: Typischer IDE-Sensor

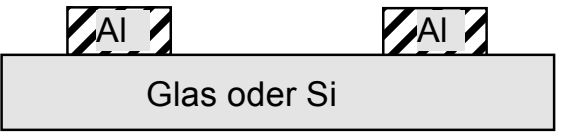

a)

SiO oder $\mathrm{SiO}_{2}, 50 \mathrm{~nm}$, (Schicht 1)

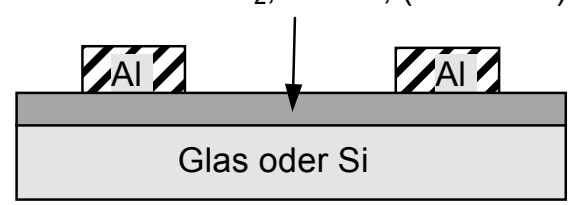

b)

Bild 1: Querschnittsgeometrie der untersuchten IDE-Elemente. a) Typ A. b) Typ B. c) Typ C. Elektrodendicke in allen Fällen $h_{\mathrm{Al}}=100 \mathrm{~nm}$.

Tabelle 1: Schichtaufbau der verwendeten Sensortypen. Die Schichten, wenn vorhanden, hatten die Dicken $h_{1}=50 \mathrm{~nm}, h_{\mathrm{Al}}=100 \mathrm{~nm}$ und $h_{2}=50 \mathrm{~nm}$.

\begin{tabular}{|l|l|l|l|l|}
\hline Sensor & Substrat & Schicht 1 & Elektroden & Schicht 2 \\
\hline A1 & Glas & - & $\mathrm{Al}$ & - \\
\cline { 2 - 5 } A2 & $\mathrm{Si}$ & - & $\mathrm{Al}$ & - \\
\hline $\mathrm{B} 1$ & $\mathrm{Glas}$ & $\mathrm{SiO}_{2}$ & $\mathrm{Al}$ & - \\
$\mathrm{B} 2$ & $\mathrm{Si}$ & $\mathrm{SiO}$ & $\mathrm{Al}$ & - \\
\cline { 2 - 5 } $\mathrm{B} 3$ & $\mathrm{Si}$ & $\mathrm{SiO}_{2}$ & $\mathrm{Al}$ & - \\
\hline $\mathrm{C}$ & $\mathrm{Glas}$ & $\mathrm{SiO}_{2}$ & $\mathrm{Al}$ & $\mathrm{SiO}_{2}$ \\
\hline
\end{tabular}

$\mathrm{SiO}_{2}, 50 \mathrm{~nm}$, (Schicht 2)

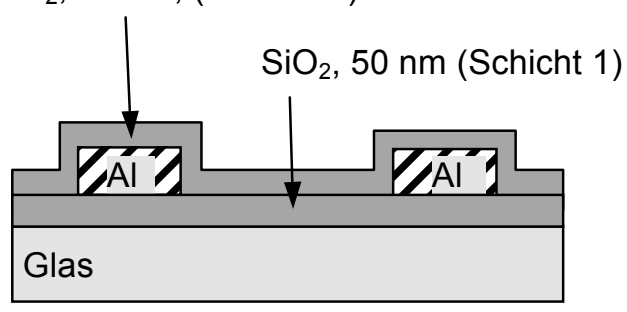

c) 


\subsection{Passivierungsschichten}

Durch Aufbringen einer Siliziumoxidschicht (Schicht 1) auf die Substratoberfläche soll die Abhängigkeit der Sensorimpedanz von der Luftfeuchtigkeit unterdrückt werden, die bei Messungen der Leerimpedanz (Sensor ohne Probe) beobachtet werden kann. Bei den in dieser Arbeit vorgestellten Experimenten sind jedoch Raumtemperatur und -feuchtigkeit so niedrig, dass die Leerimpedanz aller Sensortypen stets im Megaohmbereich liegt und damit die Luftfeuchtigkeit keine signifikante Rolle spielt.

Die Passivierungsschicht auf den Elektroden (Schicht 2) soll diese vor Beeinträchtigungen schützen, die bei Kontakt mit Gewebe (Blut, Gallenflüssigkeit, ...) eintreten könnten. Im Gegensatz zu der auf der Substratoberfläche aufgebrachten Schicht, beeinflusst die Passivierungsschicht das Messergebnis in erheblichem Maße. Um diesen Einfluss zu quantifizieren, wurde ein einfaches eindimensionales Modell der Sensoren herangezogen und damit die Impedanz der Schutzschicht abgeschätzt.

\subsection{D-Modell der Sensorgeometrie und äquivalentes Ersatzschaltbild}

Für die Experimente zur Charakterisierung der Impedanz von Leberproben wurden IDE-Sensoren jeweils komplett mit einem Gewebestück von ca. $5 \mathrm{~mm}$ Dicke bedeckt. Der Verlauf der elektrischen Feldlinien zwischen den Elektroden von Sensoren des Typs C (Bild 1) legt es nahe, die Verhältnisse zumindest näherungsweise durch ein äquivalentes eindimensionales Modell zu beschreiben (Bild 2a und [5, 6]). Dies führt zu dem elektrischen Ersatzschaltbild (ESB) in Bild 2b. Dabei geht $\underline{Z}_{\mathrm{s}}$ auf die Stromlinien im Substrat und in Schicht 1 zurück, $\underline{Z}_{p}$ auf jene in der passivierenden Schicht 2 und $\underline{Z}_{\mathrm{pL}}+k \underline{Z}_{\mathrm{L}}$ auf jene durch Schicht 2 und die Leber (siehe Bild $1 ; k=$

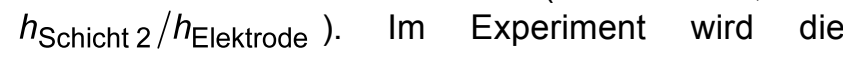
Gesamtimpedanz $\underline{Z}_{C}$ gemessen. Analoge Modelle ergeben sich für die anderen Sensortypen (Bild 2c, d).

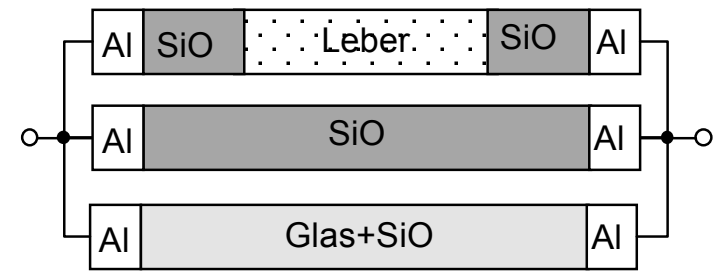

a)

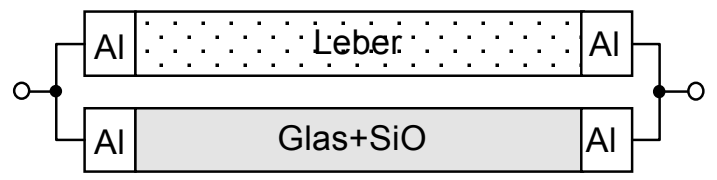

C)

Bild 2: Sensormodellierung. a) Äquivalentes 1D-Modell von Sensoren des Typs C. b) Daraus abgeleitetes äquivalentes Ersatzschaltbild. c, d) Dasselbe für Sensoren des Typs B.

Beim Feldeinsatz eines Sensors Typ C muss $\underline{Z}_{\mathrm{pL}}$ bekannt sein, damit aus der Gesamtimpedanz $\underline{Z}_{C}$ durch Deembedding die interessierende Probenimpedanz $\underline{Z}_{L}$ bestimmt werden kann. Diese Kenntnis verschafft man sich durch eine Kalibrationsmessung wie folgt. Zunächst wird die Impedanz $\underline{Z}_{B 0} \equiv \underline{Z}_{s}$ eines Sensors Typ B ohne Leberprobe gemessen, anschließend die Impedanz $\underline{Z}_{B, L}$ desselben Sensors mit Probe. Da in Abwesenheit der Leberprobe die Impedanz $\underline{Z}_{L}$ in Bild 2d durch eine kleine Kapazität ersetzt und vernachlässigt werden kann, ergibt sich für die Impedanz der Leber 


$$
\underline{Z}_{\mathrm{L}}=\frac{\underline{Z}_{\mathrm{B} 0} \underline{Z}_{\mathrm{B}, \mathrm{L}}}{\underline{Z}_{\mathrm{B} 0}-\underline{Z}_{\mathrm{B}, \mathrm{L}}} .
$$

Erfasst man analog die Leerimpedanz $\underline{Z}_{\mathrm{C} 0} \equiv \underline{Z}_{\mathrm{s}} \| \underline{Z}_{\mathrm{p}}$ und die Impedanz mit Leberprobe $\underline{Z}_{\mathrm{C}, \mathrm{L}}$ für einen Sensor des Typs $C$, lässt sich aus den Messwerten schließlich $\underline{Z}_{\mathrm{pL}}$ berechnen:

$$
\underline{Z}_{\mathrm{pL}}=\frac{\underline{Z}_{C, L}\left(\underline{Z}_{C 0}+k \underline{Z}_{L}\right)-\underline{Z}_{C 0} k \underline{Z}_{L}}{\underline{Z}_{C 0}-\underline{Z}_{C, L}}
$$

\section{Messungen}

Um die Auswirkungen von Einflussgrößen wie der Temperatur der Gewebeprobe oder des Drucks auf das Messobjekt zu studieren und um die Wirkung einer Passivierungsschicht auf den Interdigitalelektroden zu testen, wurden Impedanzspektren bei Frequenzen von $10 \mathrm{~Hz}$ bis $5 \mathrm{kHz}$ aufgenommen (Impedanzspektrometer Meodat Impspec) und dabei die Sensorantworten auf unterschiedliche Gewebesorten registriert.

Gemessen wurde mit einem Multisinusverfahren, bei dem das Messobjekt mit einem künstlichen Rauschen angeregt wird, das die gewünschten Frequenzanteile enthält. Der Vorteil dieser Methode gegenüber einem Einzelsinusverfahren ist die stark herabgesetzte Messdauer, aber es setzt voraus, dass das Messobjekt linear und zeitinvariant ist.

\subsection{Probenpräparation}

Als Proben wurden handelsübliche Rinderleber, Schweineleber und Schweinemuskelfleisch verwendet. Die Proben waren zum Zeitpunkt der Messung seit Schlachtung einen Tag alt und wurden bei $8{ }^{\circ} \mathrm{C}$ gelagert. Die Experimente wurden bei Raumtemperatur durchgeführt. Zur Applikation auf dem Sensor wurden die Gewebe zu ca. $5 \mathrm{~mm}$ dicken Quadern geschnitten, die die aktive Sensorfläche komplett bedeckten. Darüber hinaus wurden keine weiteren Präparationsschritte wie Abtupfen o. ä. vorgenommen.

\subsection{Experimente zu Störeinflüssen}

Da die Sensoren eine kapazitive Struktur aufweisen, liegt es nahe, die Bauteilimpedanz als RC-Glied zu modellieren. Allerdings lassen sich die verteilten Effekte in der dreidimensionalen Struktur nicht durch konstante konzentrierte Bauelemente wiedergeben, sondern führen auf einen frequenzabhängigen Widerstand $R(f)$ und eine ebenso frequenzabhängige Kapazität $C(f)$ (Bild 2). Es gilt dann:

$$
\underline{Z}(f)=\frac{R(f)}{1+\mathrm{j} 2 \pi f R(f) C(f)} .
$$

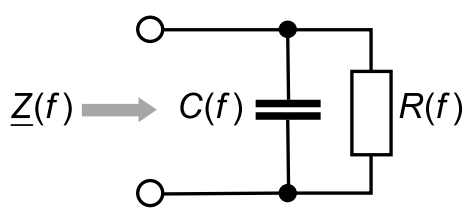

Bild 2: Darstellung der Impedanz durch ein $R C$ Glied.

Die Messungen zeigen, dass $R$ in guter Näherung von einer Potenz der Frequenz abhängt, daher wird bei der Wiedergabe der Messergebnisse eine doppelt logarithmische Darstellung gewählt.

Zur Interpretation eines Messergebnisses muss bekannt sein, welcher Anteil auf die Probe zurückzuführen ist und welcher den Sensor selbst repräsentiert. Die Sensorantwort hängt außerdem von der Temperatur, dem Eigengewicht der Probe, der vorherigen Lagerung und anderen Einflussgrößen ab. Auf solche möglichen Einflussgrößen wird im Folgenden eingegangen.

\subsubsection{Vergleich verschiedener Sensortypen}

Bild 3 zeigt die Leerimpedanz von vier Strukturen: Sensor A2 mit der Schichtfolge Si|Al, Sensor B1 $\left(\mathrm{Glas}\left|\mathrm{SiO}_{2}\right| \mathrm{Al}\right)$, Sensor B2 (Si|SiO|Al) und Sensor $\mathrm{C}\left(\mathrm{Glas}\left|\mathrm{SiO}_{2}\right| \mathrm{All} \mid \mathrm{SiO}_{2}\right)$. Die auf dem halbleitenden Siliziumsubstrat aufgebauten Sensoren weisen deutlich niedrigere Widerstandswerte und deutlich höhere Kapazitäten auf als die Sensoren auf Glas. Bei allen Sensoren sinken die Widerstandswerte mit steigender Frequenz, die Kapazität hingegen bleibt konstant. Dies ist das typische Verhalten von Bauelementen, die Schottkykontakte an den Elektroden oder Grenzflächen zu Festkörper-lonenleitern aufweisen, wie es hier der Fall ist [5]. Vergleicht man die Leerimpedanzen mit den Impedanzen bei aufgelegter Rinderleber (Bild 4), so wird klar, dass der Verlauf der Widerstandswerte allein durch den 


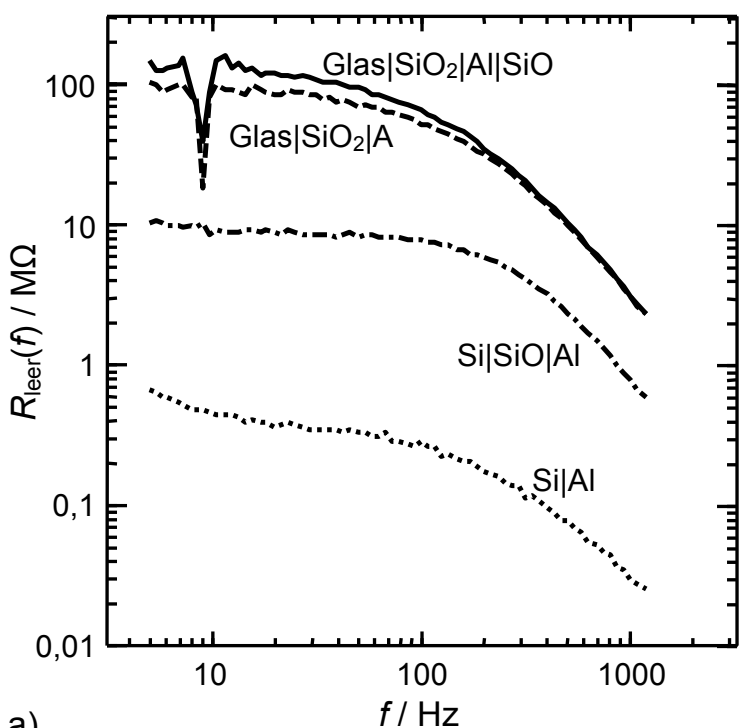

a)

Bild 3: Gemessene Leerimpedanz verschiedener Sensoren als Funktion der Frequenz. a) Parallelwiderstand $R_{\text {leer }}$, b) Parallelkapazität $C_{\text {leer }}$.

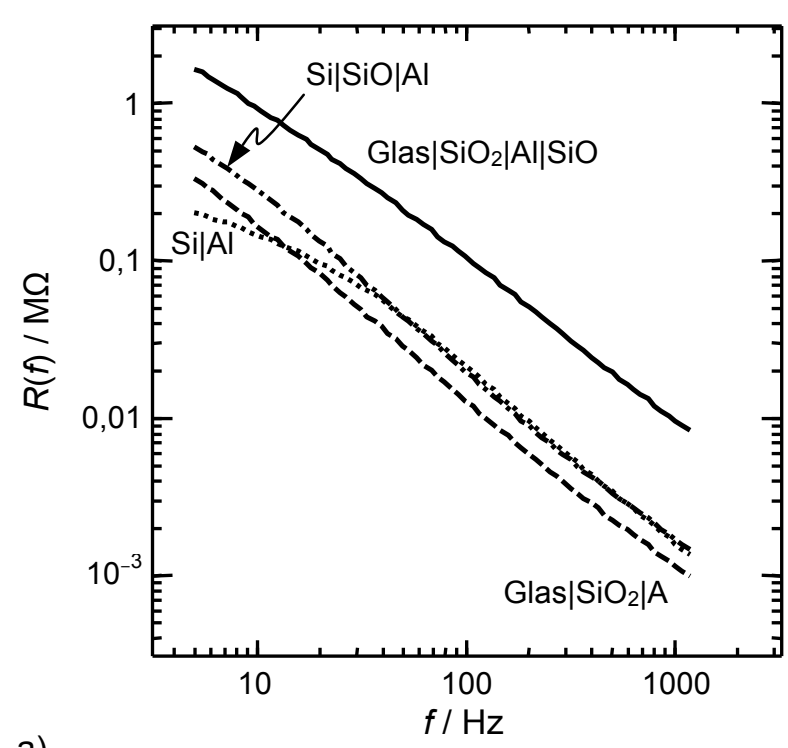

a)

Bild 4: Gemessene Sensorimpedanz bei Kontakt mit Rinderleber. a) Parallelwiderstand $R$. b) Parallelkapazität $C$.

Pfad durch die Leber bestimmt wird (vgl. Bild 1). Das Beispiel des Sensors C $\left.\left(\mathrm{Glas}\left|\mathrm{SiO}_{2}\right| \mathrm{Al} \mid \mathrm{SiO}\right)_{2}\right)$ verdeutlicht, dass eine Beschichtung der Elektroden den absoluten Wert der Messung beeinflusst; zur Extraktion der wahren Leberimpedanz ist das unter 2.2 beschriebene Deembedding nötig.

Die geringen Unterschiede zwischen den Widerstandswerten für die Sensoren B1 und B2 sind möglicherweise auf eine spontan gebildete Oxidhaut auf den Aluminiumelektroden zurückzuführen. Auffällig ist die sehr gute Parallelität der Messkurven, die Steigung der Kurven scheint daher ein gut reproduzierbares Charakteristikum eines individuellen Lebergewebes darzustellen.

Wiederum beeinflusst die Passivierungsschicht des Sensors C das Messergebnis, da diese eine Serienkapazität zur Leberkapazität bildet und damit den Messwert der Parallelkapazität erniedrigt. Die besten Eigenschaften zeigt Sensor B1 (Schichtfolge $\mathrm{Glas}\left|\mathrm{SiO}_{2}\right| \mathrm{Al}$ ). Bei ihm ist die Leerimpedanz sehr groß, was gegenüber den Sensoren auf Silizium eine höhere Empfindlichkeit bewirkt. 


\subsubsection{Charakterisierung der Passivierungsschicht}

In Bild 5 ist das Ergebnis einer Kalibration dargestellt, die wie in Abschnitt 2.2 beschrieben das Ziel hat, den Einfluss der Elektrodenpassivierung zu erfassen. Die nur $50 \mathrm{~nm}$ dicke Passivierungsschicht wirkt sich, wie behauptet, erheblich auf das Messergebnis aus. Somit liegt die Vermutung nahe, dass auch natürliche Oxidschichten, wie sie sich z. B. auf Aluminiumelektroden stets spontan bilden, eine Messung beeinflussen und bei längerer Lagerung der Sensoren Alterungseffekte eintreten.
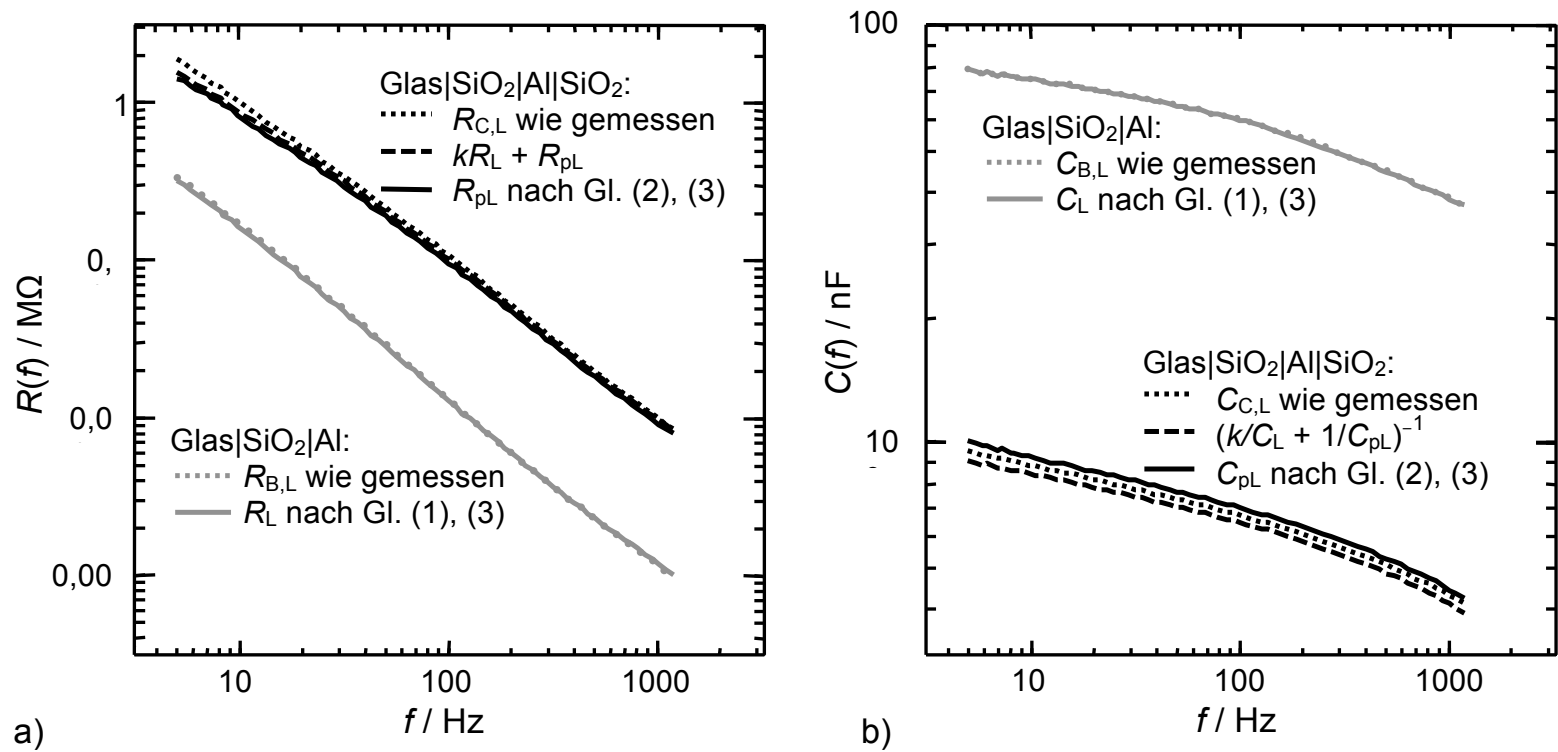

Bild 5: Ergebnis einer Kalibrationsmessung zur Bestimmung der Passivierungsschichtimpedanz $\underline{Z}_{\mathrm{pL}}$ (Verfahren nach Abschnitt 2.2 mit $k=0,5$ ). a) Parallelwiderstand $R$. b) Parallelkapazität $C$.

\subsubsection{Temperatureinfluss}

Legt man gekühlte Leberproben $\left(\vartheta_{1} \approx 16^{\circ} \mathrm{C}\right)$ auf einen Sensor und lässt sie sich danach auf Raumtemperatur $\left(\vartheta_{3} \approx 22^{\circ} \mathrm{C}\right)$ erwärmen, so beobachtet man, dass unabhängig vom Sensortyp der Parallelwiderstand $R(f)$ mit der Zeit, also mit der Temperatur, sinkt und die Parallelkapazität $C(f)$ steigt (Bild 6). Dies lässt sich auf die mit der Erwärmung steigende Beweglichkeit der Ladungsträger (Ionen) in der Leber zurückführen.

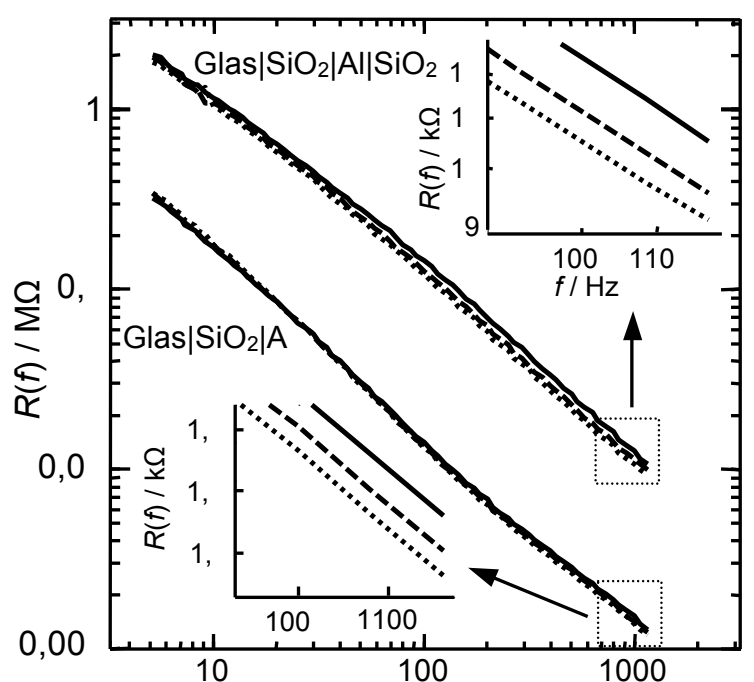

a)

Bild 6: Gemessener Temperatureinfluss auf a) den Parallelwiderstand $R(f)$ und b) die Parallelkapazität $C(f)$ einer Rinderleberprobe auf einem Sensor $C\left(\mathrm{Glas}\left|\mathrm{SiO}_{2}\right| \mathrm{Al} \mid \mathrm{SiO}_{2}\right)$ und einem Sensor $\mathrm{B} 1\left(\mathrm{Glas}\left|\mathrm{SiO}_{2}\right| \mathrm{Al}\right) .-\mathrm{V}: \vartheta_{1} \approx 16^{\circ} \mathrm{C} ; \boldsymbol{-}^{---}: \vartheta_{2}\left(\vartheta_{1}<\vartheta_{2}<\vartheta_{3}\right) ; \cdots \cdot \cdot \vartheta_{3} \approx 22{ }^{\circ} \mathrm{C}$.

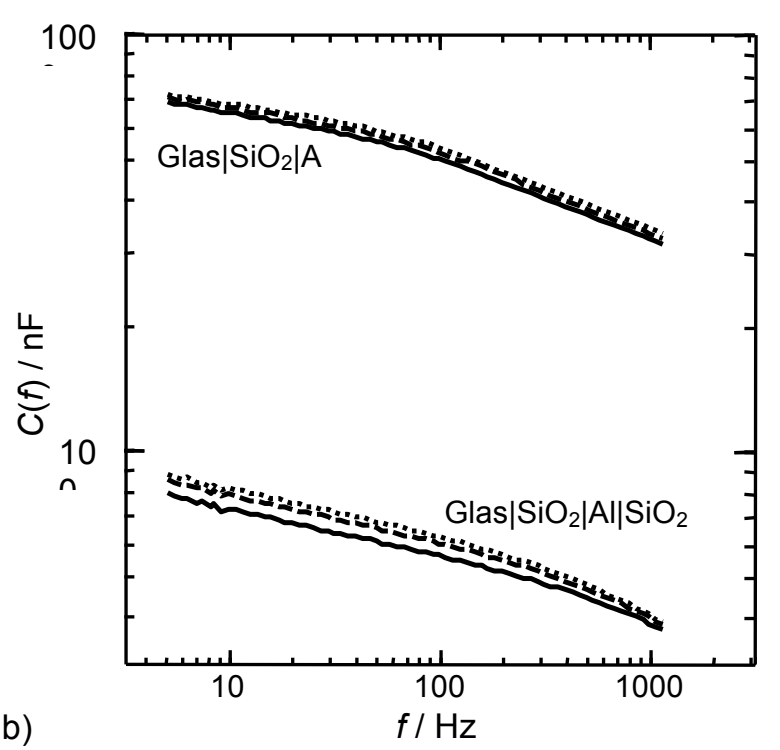

\footnotetext{
b)
} 


\subsubsection{Druckeinfluss}

Unter der Annahme, dass auf Grund des Feldlinienverlaufs in der Probe die gemessene Sensorimpedanz nur von einer dünnen Schicht der Probe beeinflusst wird, spielt deren genaue Geometrie nur eine untergeordnete Rolle. Dagegen sollte das Eigengewicht der Gewebeprobe oder ein Anpressdruck an die Sensoroberfläche die Messung beeinflussen. Um dies zu untersuchen, wurde zunächst ein leichter Anpressdruck auf die zu vermessende Rinderleberprobe ausgeübt, wodurch diese sich elastisch verformt. Etwa $60 \mathrm{~s}$ nach Entlastung nimmt die Leber wieder ihre Ausgangsform an. Bild 7 gibt die jeweils gemessenen Impedanzen wieder (die Messung während des Entspannungsvorganges muss qualitativ interpretiert werden, da sich das Messobjekt hier während der einige Sekunden andauernden Messung ändert). Deutlich ist ein Sinken des Parallelwiderstandes $R$ und ein Anstieg der Kapazität, letzterer vor allem bei niedrigen Frequenzen, zu beobachten. Erklärbar ist dies durch die Erhöhung der Ladungsträgerdichte im Pfad durch das mechanische Zusammenquetschen der Leber und ein verbessertes Einpassen in die Zwischenräume zwischen den einzelnen Elektrodenfingern. Es zeigt sich damit, dass die Probendicke, das heißt das Eigengewicht der Leberprobe, die Messung beeinflusst.
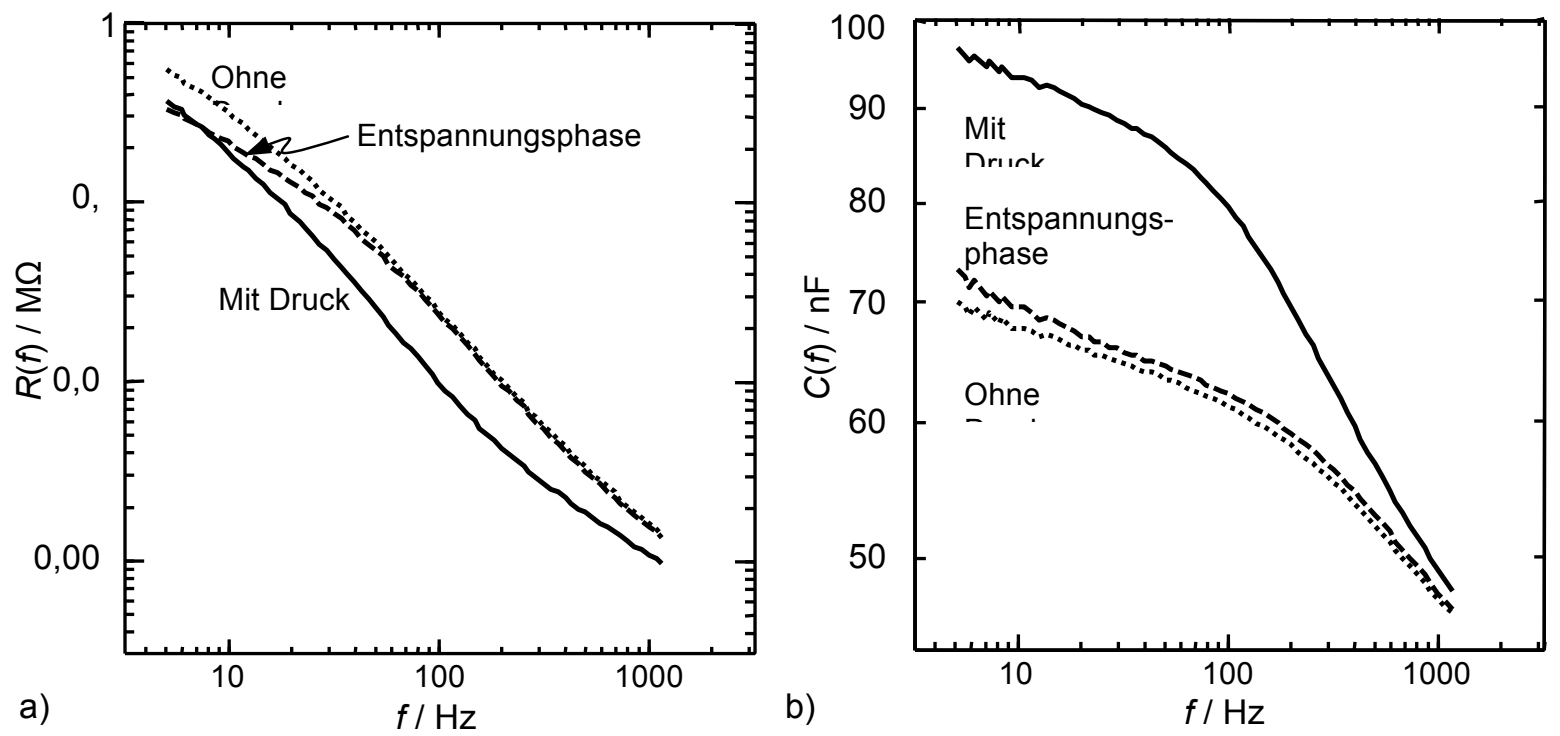

Bild 7: Gemessene Reaktion der Impedanz eines Sensors B3 $\left(\mathrm{Si}\left|\mathrm{SiO}_{2}\right| \mathrm{Al}\right)$ in Kontakt mit Leber auf den Druck, mit dem die Leber angepresst wird. a) Parallelwiderstand $R(f)$. b) Parallelkapazität $C(f)$.

\subsection{Experimentelle Charakterisierung unterschiedlicher Gewebesorten}

\subsubsection{Vergleich von Rinderleber und Schweinemuskel}

Mit einem Sensor B2 (Si|SiO|Al) wurden die Impedanzspektren zweier Proben aus derselben Rinderleber und einer Probe eines Schweinemuskelfleischs (Oberschale) aufgenommen. Dabei wurden die Fasern der Schweinemuskelprobe einmal senkrecht zu den Elektrodenfingern ausgerichtet und einmal parallel. Die Proben wurden nacheinander aufgelegt, ohne den Sensor dazwischen zu reinigen. Begonnen wurde mit den Schweinefleischproben, deren Oberfläche kaum Flüssigkeit absondert, so dass die Sensoroberfläche nur wenig verschmutzt wurde und die Sensorimpedanz nach Entfernung der Probe nahezu wieder die hohen Werte der Leerimpedanz erreichte. Die anschließenden Messungen der Leberproben verunreinigten das Elektrodenfeld erheblich mehr, da die vor der Messung frisch der Leber entnommenen Teile noch Blut und andere Flüssigkeiten absonderten (Bild 8a).

Nach dem ersten Messzyklus wurde die Sensoroberfläche durch Spülen unter fließendem kalten Wasser gereinigt, so dass die Leerimpedanz des Sensors wieder ihren Ausgangswert annahm (Bild 8b). Sofort im Anschluss folgte der zweite Messzyklus analog dem ersten, jedoch mit neuen Proben.

Die Messungen belegen, dass der betrachtete Mikrosensor zwischen Leber und Schweinemuskel unterscheiden kann, im Fall des Schweinemuskels sogar zwischen den verschiedenen Faserrichtungen (Bild 9). Bei allen Proben verschieben sich die Kapazitätskurven zwischen der ersten und zweiten Messung hin zu höheren Werten (Bild 9a, b). Ob dies die Folge des Abriebs einer dünnen Schicht (Oxidschicht?) auf den Elektroden im Laufe der ersten Messung ist, muss noch untersucht werden. 
a)

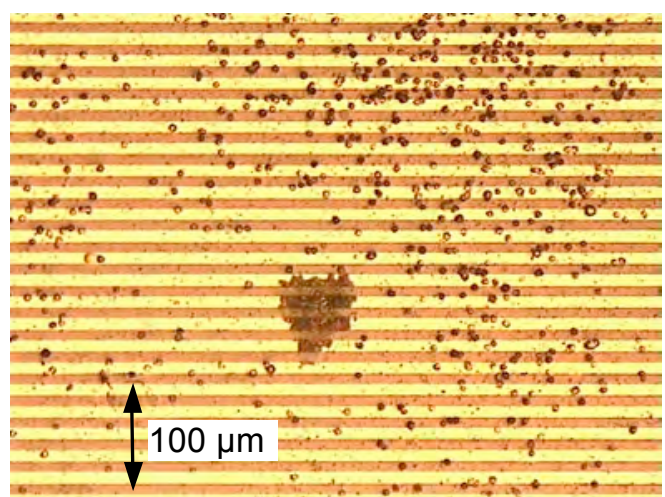

b)

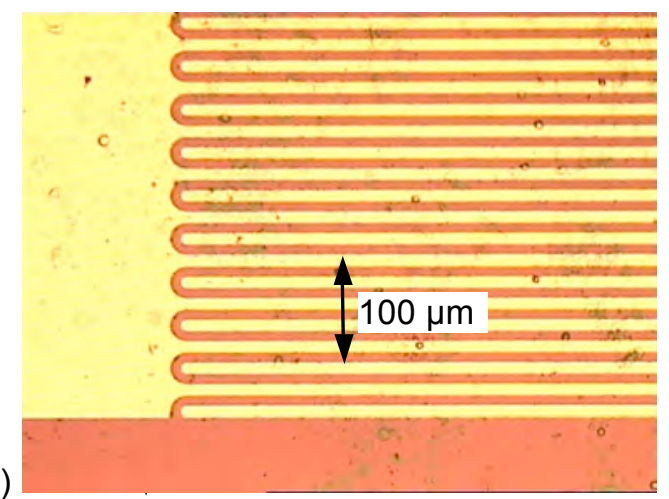

Bild 8: Mikroskopaufnahme der Oberfläche eines Sensors B2 (Si|SiO|AI). a) Nach Verunreinigung mit Erythrozyten. b) Nach Reinigung.
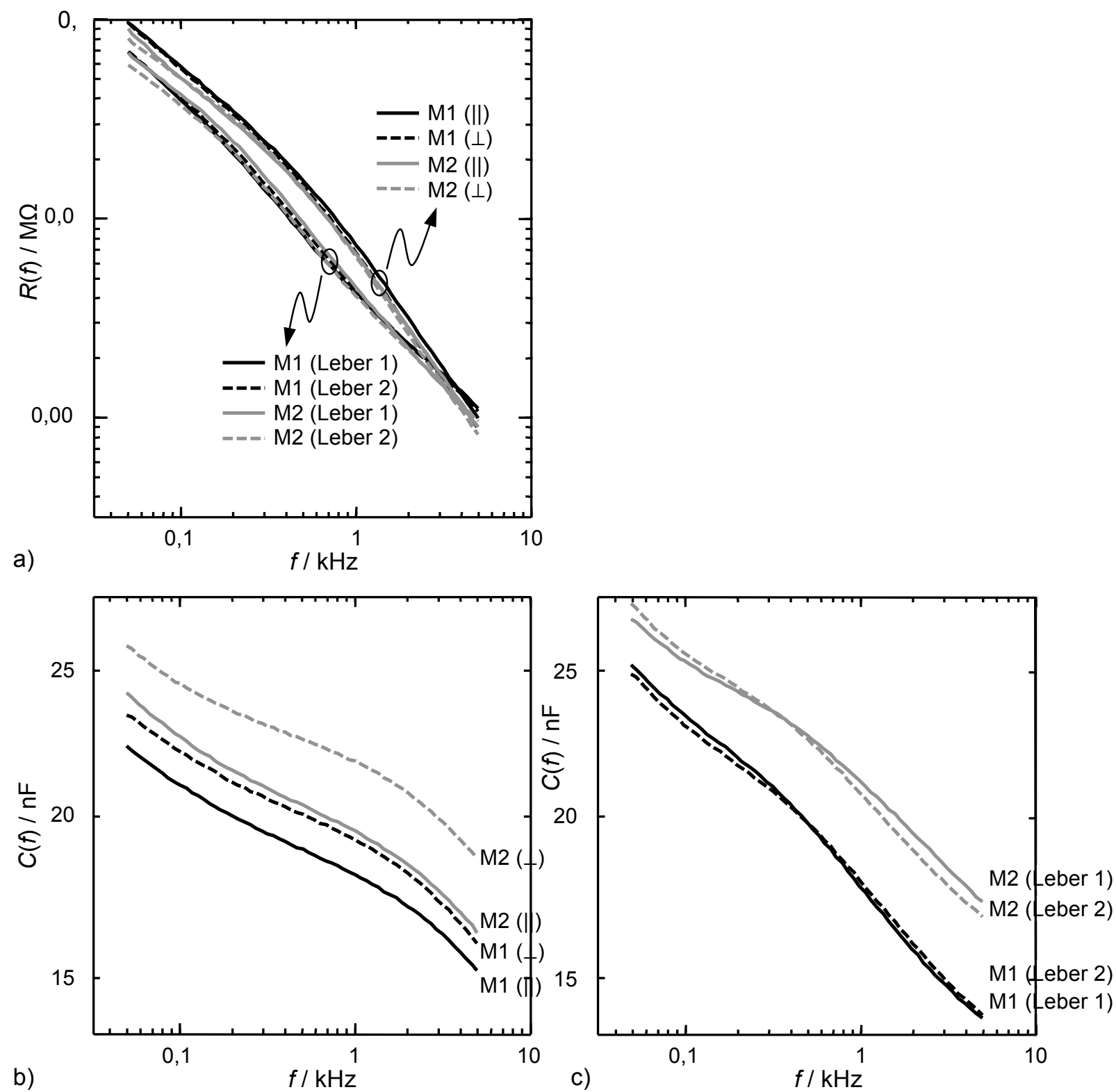

Bild 9: Sensorimpedanz bei verschiedenen Gewebesorten. Erste Messung (M1): frischer Sensor B2 (Si|SiO|Al); zweite Messung (M2): Sensor B2 nach Reinigung mit kaltem Leitungswasser. a) Parallelwiderstand $R(f)$ bei Rinderleber und Schweinemuskel. b) Parallelkapazität $C(f)$ mit Schweinemuskel. c) $C(f)$ mit Rinderleber. 


\subsubsection{Rinderleber verletzungsfrei und nicht verletzungsfrei}

Soll eine Leber verletzungsfrei untersucht werden, muss der Sensor von außen auf das Bindegewebe (Epithel) gelegt werden, das die Leber umgibt. Erste Messungen belegen, dass das Epithel das Impedanzspektrum der Leberprobe in seiner Kurvenform deutlich verändert (Bild 10). Da diese Leberhaut die Sensoroberfläche vor den Flüssigkeiten der Leber schützt, kann das Messergebnis bei mehrmaligem Auflegen der Probe auch ohne Reinigung des Sensors sehr gut reproduziert werden.
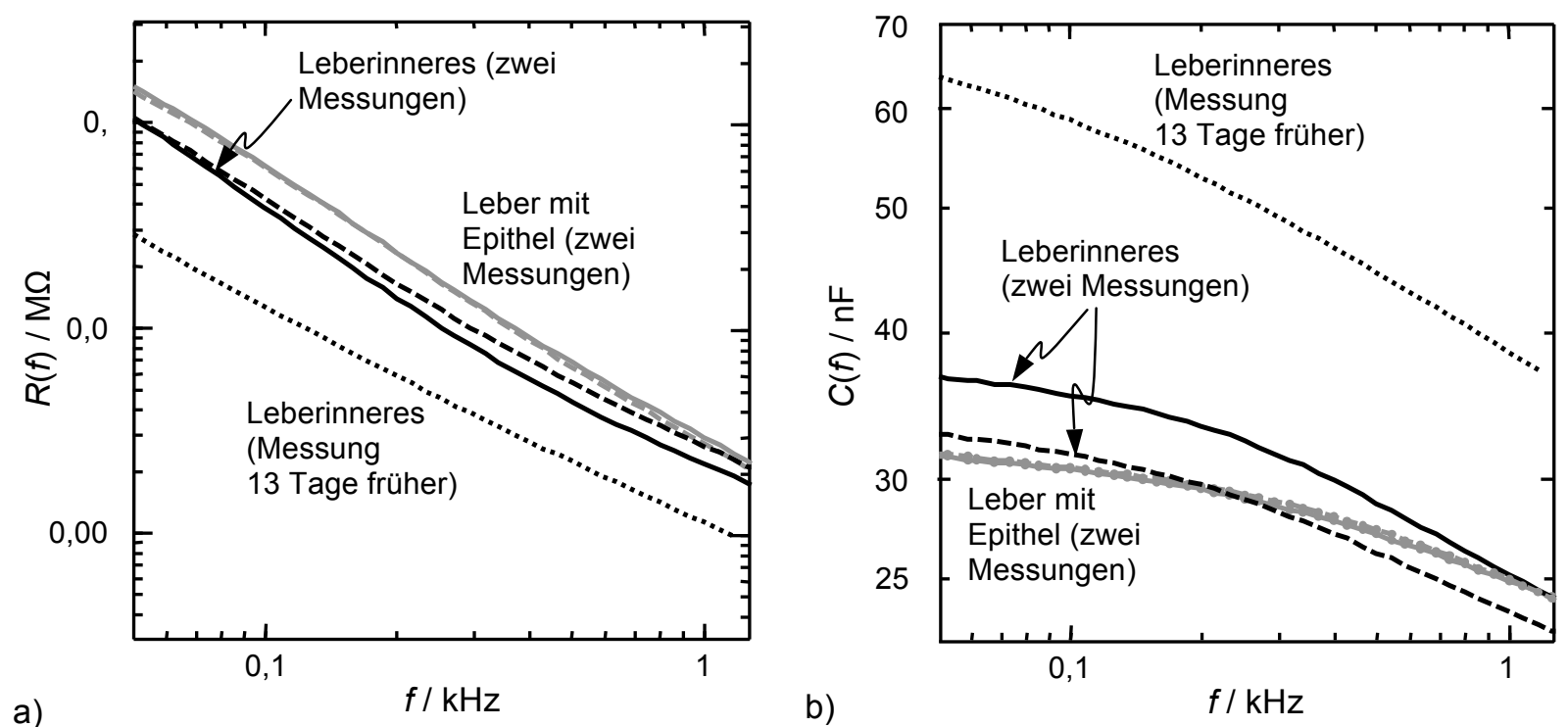

Bild 10: Messung einer Leberprobe aus dem Inneren einer frischen Rinderleber und einer Probe mit Leberepithel mit einem Sensor B1 $\left(\mathrm{Glas}\left|\mathrm{SiO}_{2}\right| \mathrm{Al}\right)$. Zum Vergleich die frühere Messung einer Probe aus dem Inneren einer frischen Rinderleber mit demselben Sensorexemplar, die zeigt, dass sich in der Zwischenzeit eine Oxidhaut auf den Elektroden gebildet haben muss. a) Parallelwiderstand $R(f)$. b) Parallelkapazität $C(f)$.

\subsubsection{Alterung von Schweineleber}

Wird eine Leber vom Blutkreislauf getrennt, endet die Sauerstoffversorgung und verschiedene Zersetzungsprozesse beginnen. Die Leberzellen können eine Zeitlang überleben, indem sie durch anaerobe Glykolyse Energie gewinnen. Die dabei entstehende Säure lässt den pH-Wert im Gewebe sinken, was die Anzahl der zum elektrischen Ladungstransport bereitstehenden lonen verändert. Gleichzeitig breiten sich Bakterien aus [1,7]. Um zu testen, ob diese Prozesse sich im Impedanzspektrum beobachten lassen, wurde eine handelsübliche Schweineleber wie in Tabelle 2 beschrieben über drei Tage hinweg impedimetrisch mit einem Sensor A1 (Glas|Al) untersucht (Bild 11).

Alle Gewebeteile wurden zwischen den Messungen bei etwa $8{ }^{\circ} \mathrm{C}$ gelagert. Die Impedanz der separat gelagerten Probe 1 änderte sich im Laufe der drei Tage nur wenig. Offenbar verhinderte die separate Lagerung den Kontakt mit den von der Leber abgesonderten Flüssigkeiten, die ein Bakterienwachstum begünstigen. Auch die am zweiten Tag entnommene Probe zeigt am selben Tag noch in etwa dasselbe Impedanzniveau und einen ähnlichen Kurvenverlauf wie die Probe 1 vom Vortag, die den frischen Ausgangszustand repräsentiert. Erst am dritten Tag ändern sich die Impedanzwerte der Proben 2 und 3 um ein bis zwei Größenordnungen, und der Frequenzgang der Parallelkapazität ändert sich signifikant. Der Verfallsprozess spiegelt sich also deutlich im Impedanzspektrum des Messobjektes „Leber" wieder.

Tabelle 2: Alterungsuntersuchungen an einer Schweineleber an drei Tagen nach der Schlachtung.

\begin{tabular}{|l|l|}
\hline Tag & Aktion \\
\hline 1 & $\begin{array}{l}\text { a) Messung einer Probe aus dem Leberinneren } \\
\text { b) Lagerung von Probe 1 getrennt von der restlichen Leber }\end{array}$ \\
\hline 2 & $\begin{array}{l}\text { a) Erneute Messung von Probe 1, weitere getrennte Lagerung } \\
\text { b) Entnahme und Messung einer zweiten Probe aus dem Leberinneren } \\
\text { c) Lagerung von Probe } 2 \text { mit der restlichen Leber }\end{array}$ \\
\hline 3 & $\begin{array}{l}\text { a) Erneute Messung der Proben 1 und 2 } \\
\text { b) Entnahme und Messung einer dritten Probe aus dem Leberinneren }\end{array}$ \\
\hline
\end{tabular}




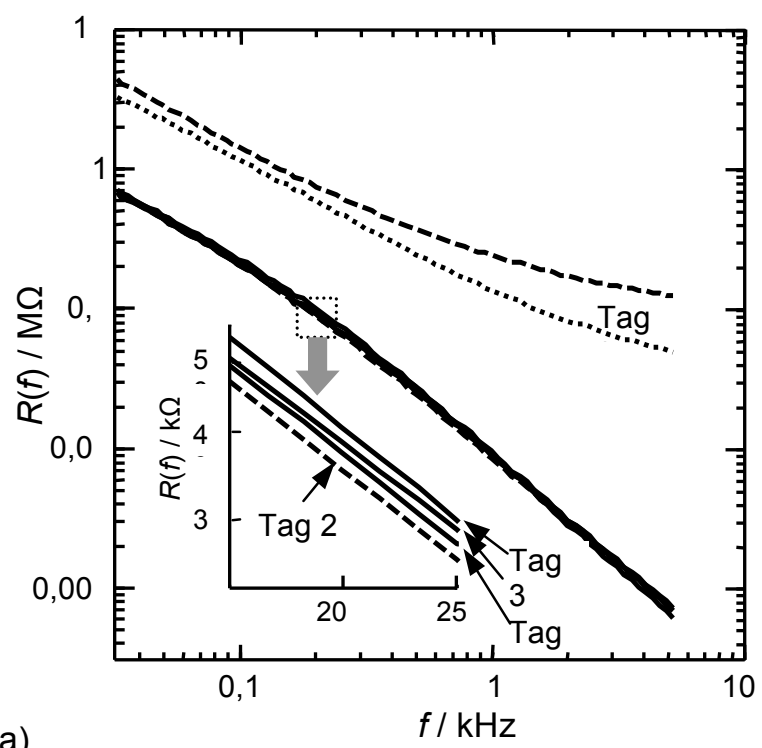

a)

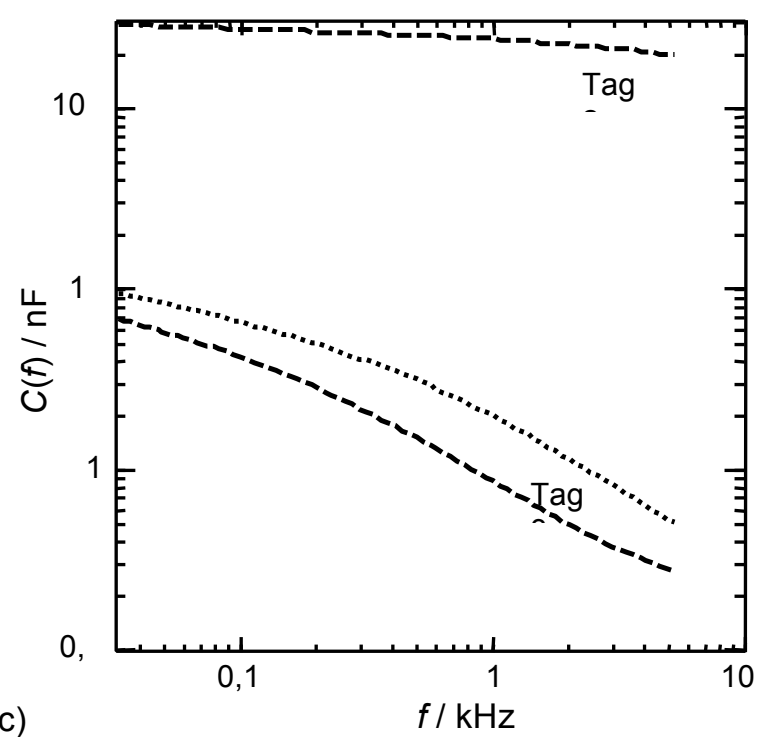

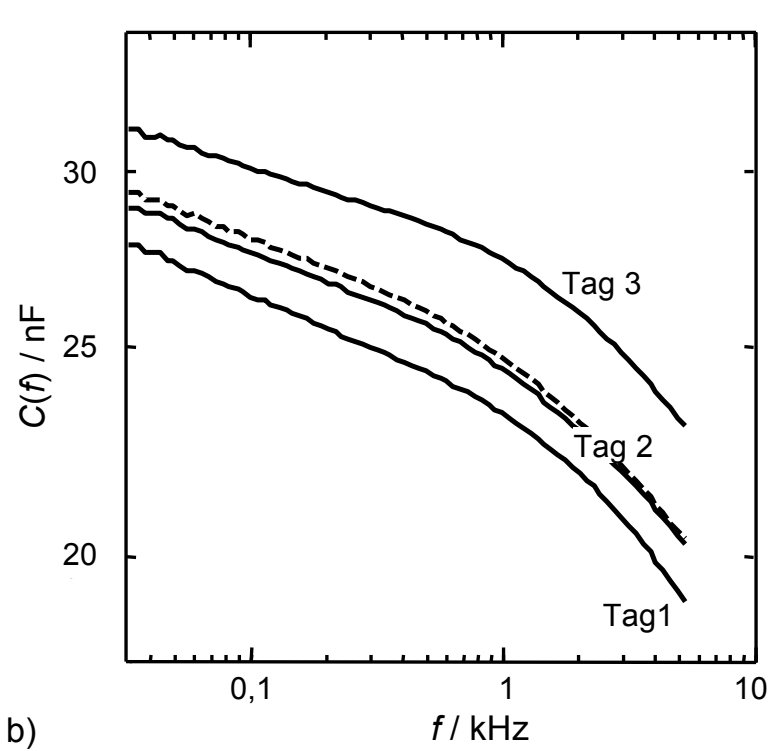

Bild 11: Alterung von Schweineleberproben über drei Tage, beobachtet mit einem Sensor A1 (Glas|Al). - Probe 1; --- : Probe 2; '..... Probe 3. a) Parallelwiderstand $R(f)$. b, c) Parallelkapazität $C(f)$.

\section{Zusammenfassung}

In dieser Arbeit wurde evaluiert, ob planare IDE-Chemosensoren zur Charakterisierung von Gewebe im allgemeinen und von Leber im besonderen herangezogen werden können. Dabei wurde der Einfluss des Sensoraufbaus, der Einflussgrößen Temperatur und Druck und einer Passivierungsschicht auf den Elektroden auf das Messergebnis untersucht. Messungen an verschiedenartigen Gewebeproben zeigen die Fähigkeit des Sensors zur Unterscheidung zwischen den Gewebesorten. Am Beispiel einer Schweineleber konnte gezeigt werden, dass der Sensor auf die Alterung des Gewebes nach der Schlachtung anspricht.

Die durchgeführten Experimente belegen, dass impedimetrische IDE-Sensoren auf Grund der hohen Informationsdichte des gewonnen Messergebnisses das Potential zu einer detaillierten Charakterisierung von Geweben besitzen. Für quantitative Aussagen zu den Materialeigenschaften der vermessenen Gewebeprobe (Permittivität, Leitfähigkeit) müssen jedoch die Messbedingungen und die Schichtenfolge des Sensors (Passivierungsschicht, spontane Oxidhaut) genau bekannt sein. 


\section{Literatur}

[1] J. Harms, A. Schneider, M. Baumgartner, J. Henke, R. Busch, „Diagnosing acute liver graft rejection: experimental application of an implantable telemetric impedance device in native and transplanted porcine livers“, Biosensors and Bioelectronics 16 (2001), S. 169-177.

[2] Y. Chuang, Y. Chang, K. Liu, H. Chang, T. Yew, „Electrical impedance biosensors for liver function detection", Biosensors and Bioelectronics 28 (2011), S. 368-372.

[3] T. Yamada, H. Hirose, Y. Mori, E. Sasaki, A. Onitsuka, S. Senga, N. Futamura, K. Sakamoto, T. Sago, M. Yasumura, H. Iwata, „An Experimental Estimation of the Maximum Period of Liver Preservation Using Dielectric Parameters“, Transplantation Proc. 34 (2002), S. 1098-1104.

[4] U. Tröltzsch, O. Kanoun, M. Arnold, Ch. Stöckel, „Untersuchungen zur Machbarkeit von Fleischqualitätsbewertung mit Impedanzspektroskopie“, Proc. Sensoren und Messsysteme, Nürnberg (18.19. Mai 2010), S. 134-139.

[5] A. Fischerauer, G. Fischerauer, „Physikalisches Modell der Materialparameterabhängigkeit des Impedanzspektrums planarer Chemosensoren in Mehrschichtbauweise“, Technisches Messen 78 (2011) 1, S. 15-22.

[6] A. Fischerauer, G. Fischerauer, „Modellgestützte Parameteridentifikation für Impedanzspektren planarer Chemosensoren", Proc. Sensoren und Messsysteme, Nürnberg (18.-19. Mai 2010), S. 124-129.

[7] E. Gersing, „Impedance spectroskopy on living tissue for determination of the state of organs“, Biosensors and Bioelectronics 45 (1998), S. 145-149. 